\title{
Determination of Upconversion Quantum Yields Using Charge- Transfer State Fluorescence of Heavy-Atom-Free Sensitizer as a Self-Reference
}

\author{
Natalia Kiseleva \\ Karlsruhe Institute of Technology \\ Dmitry Busko \\ Karlsruhe Institute of Technology \\ Bryce Richards \\ Karlsruhe Institute of Technology
}

See next page for additional authors

Follow this and additional works at: https://arrow.tudublin.ie/scschcpsart

Part of the Chemistry Commons

\section{Recommended Citation}

Kiseleva, N., Busko, D., Richards, B.S., Filatov, M.A., Turshatov (2020). Determination of upconversion quantum yields using charge-transfer state fluorescence of heavy-atom-free sensitizer as a self-reference. The Journal of Physical Chemistry Letters11,XXX., p.6560-6566. doi:10.1021/acs.jpclett.0c01902

This Article is brought to you for free and open access by the School of Chemical and Pharmaceutical Sciences at ARROW@TU Dublin. It has been accepted for inclusion in Articles by an authorized administrator of ARROW@TU Dublin. For more information, please contact arrow.admin@tudublin.ie, aisling.coyne@tudublin.ie, gerard.connolly@tudublin.ie.

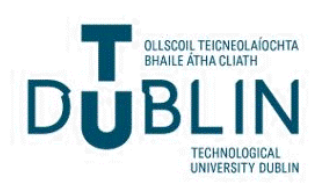


Authors

Natalia Kiseleva, Dmitry Busko, Bryce Richards, Mikhail Filatov, and Andrey Turshatov

This article is available at ARROW@TU Dublin: https://arrow.tudublin.ie/scschcpsart/99 


\section{Determination of Upconversion Quantum Yields}

\section{using Charge-Transfer State Fluorescence of}

\section{Heavy-Atom-Free Sensitizer as a Self-Reference}

Natalia Kiseleva, ${ }^{\mathrm{a}}$ Dmitry Busko, ${ }^{\mathrm{a}}$ Bryce S. Richards, ${ }^{\mathrm{a}, \mathrm{b}}$ Mikhail A. Filatov, ${ }^{\mathrm{c}}$ Andrey Turshatov $^{\mathrm{a}}$

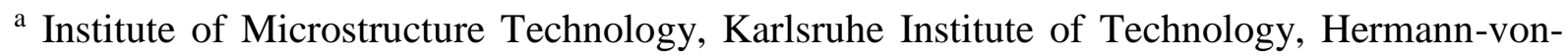
Helmholtz-Platz 1, 76344, Eggenstein-Leopoldshofen, Germany,

${ }^{\mathrm{b}}$ Light Technology Institute, Karlsruhe Institute of Technology, Engesserstrasse 13, 76131 Karlsruhe, Germany.

${ }^{c}$ School of Chemical and Pharmaceutical Sciences, Technological University Dublin, City Campus, Kevin Street, Dublin 8, Ireland

AUTHOR INFORMATION

Corresponding Author

*Mikhail A. Filatov mikhail.filatov@ tudublin.ie; Andrey Turshatov andrey.turshatov@ kit.edu 
ABSTRACT: The efficiency of photon upconversion via triplet-triplet annihilation is characterized by an upconversion quantum yield $\left(\Phi_{U C}\right)$, however there remains uncertainties for its determination. Here, we present a new approach for the relative measurement of $\Phi_{U C}$ for green-to-blue upconversion using BODIPY-pyrene donor-acceptor dyad (BD1) as a heavyatom-free triplet sensitizer. This new approach exploits broad fluorescence from a chargetransfer (CT) state of BD1, which possesses: i) a significant Stokes shift of $181 \mathrm{~nm}$ in dichloromethane; and ii) a comparably-high CT-fluorescence quantum yield ( $\left.\Phi_{\text {ref }}=7.0 \pm 0.2 \%\right)$, which is independent from oxygen presence and emitter (perylene) concentration while also exhibiting a linear intensity dependence. Based on this, we developed an upconversion reference using the BDI sensitizer mixed with perylene $\left(1 \times 10^{-5} \mathrm{M} / 1 \times 10^{-4} \mathrm{M}\right)$ in dichloromethane. With this reference system, we investigated the performance of three BODIPY donor-acceptor dyads in the upconversion process and achieved one of the highest $\Phi_{U C}$ of $6.9 \pm 0.2 \%$ observed for heavy-atom-free sensitizers to date.

\section{TOC GRAPHIC}

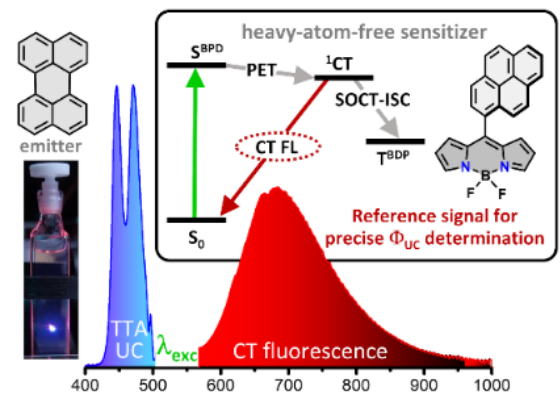


Photon upconversion (UC) via the mechanism of triplet-triplet annihilation (TTA) can be performed with high upconversion quantum yield $\left(\Phi_{U C}\right)$ under excitation with low-cost lightemitting diodes or laser sources of moderate excitation intensity $\left(\mathrm{mW} / \mathrm{cm}^{2}-\mathrm{W} / \mathrm{cm}^{2}\right.$ range). ${ }^{1}$ Due to this unique feature TTA-UC has found various applications in such areas as photocatalysis, ${ }^{2-4}$ solar energy conversion,,$^{5-6}$ bioimaging $^{7}$ and photomedicine. ${ }^{8}$ As illustrated in Figure 1a, a typical TTA-UC system combines two components - a sensitizer and an emitter, undergoing a series of photophysical processes: population of the lowest singlet excited state of a sensitizer, intersystem crossing (ISC), triplet-triplet energy transfer (TTET), triplet-triplet annihilation (TTA), and radiative relaxation of a singlet excited state of the emitter.

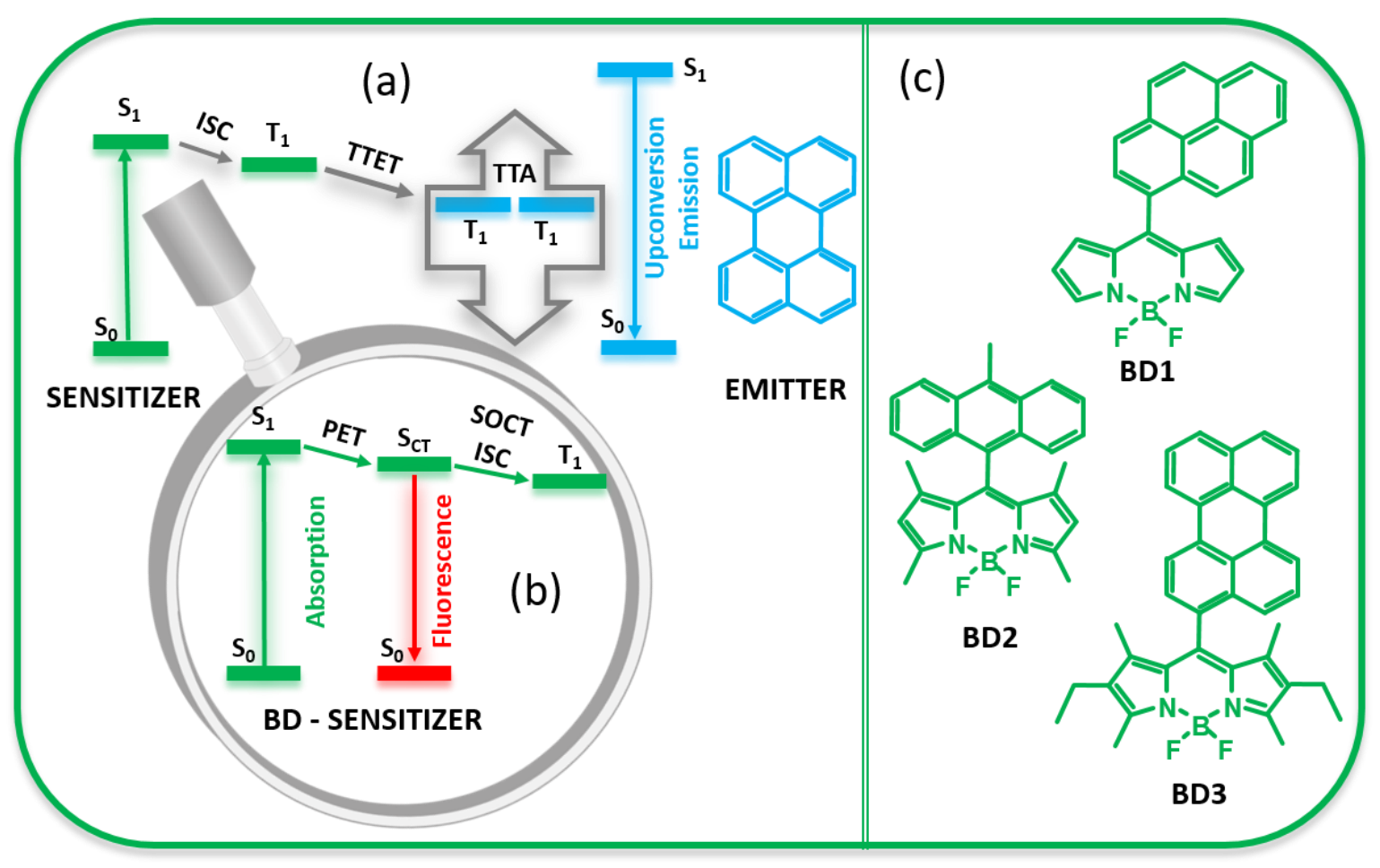

Figure 1. Generalized Jablonski diagram illustrating the TTA-UC process for: (a) standard sensitizers containing heavy atoms and (b) for heavy-atom-free sensitizers producing triplet excited states via charge transfer; (c) structures of the three BODIPY donor-acceptor dyads (BD1-3) investigated in this paper as heavy-atom-free sensitizers. 
Common sensitizers employed in TTA-UC are halogenated dyes, transition metal complexes of porphyrins and other heterocyclic ligands. ${ }^{9}$ The formation of triplet excited states in such compounds takes place via spin-orbital interaction promoted by heavy atoms (transition metals and halogens). ${ }^{10}$ Due to the growing interest in TTA-UC and other processes relying on triplet state formation, alternative approaches for promoting ISC in heavy-atom-free organic molecules are being actively investigated, such as radical-enhanced ISC, ${ }^{11-12}$ twist-induced ISC $^{13}$ and charge-transfer (CT) state recombination. ${ }^{14-15}$ The latter mechanism was observed for various donor-acceptor dyad molecules and proceeds via the so-called spin-orbit charge transfer intersystem crossing (SOCT-ISC). ${ }^{16}$ Several types of dyads operating via this mechanism, for example BODIPY-anthracenes ${ }^{17-18}$ and BODIPY-perylenes ${ }^{19}$, have been employed in green-to-blue TTA-UC and demonstrated $\Phi_{U C}$ values of up to $15.8 \%,{ }^{17}$ which is comparable with corresponding halogenated BODIPY derivatives.

The structures of BODIPY donor-acceptor dyads BD1-3 employed in this work as sensitizers for green-to-blue UC and the pathway for triplet excited states formation in corresponding TTA-UC systems are illustrated in panels $\mathbf{c}$ and $\mathbf{b}$ of Figure1, respectively. Photoinduced electron transfer (PET) from the donor subunit of the dyad (pyrene in case of BD1) to the acceptor subunit (BODIPY) results in formation of a CT state, which further produces BODIPY triplet excited state via the SOCT-ISC process. ${ }^{20}$

As for any other photoluminescence process, a key parameter for TTA-UC system is $\Phi_{U C}$, defined by IUPAC as the ratio between a number of photons emitted to a number of photons absorbed by the system ${ }^{21}$. Since TTA-UC is a bimolecular process in which two absorbed low energy photons are required to produce one photon of higher energy, the maximum theoretical value of $\Phi_{U C}$ cannot exceed $50 \%$. For this reason, experimentally obtained photoluminescence quantum yields for TTA-UC systems are often multiplied by a factor of two (in order to set the maximum value of $\Phi_{U C}$ to $100 \%$ ), however this non-standard practice led to discrepancies in 
the published values of $\Phi_{U C}$ for similar TTA-UC systems. ${ }^{22}$ To avoid such discrepancies in our report, we followed the IUPAC definition and did not apply this doubling in our calculations of $\Phi_{U C}$.

Two experimental methods are commonly used for the determination of $\Phi_{U C}$ :

1. The first method is known as an absolute quantum yield measurement using an integrating sphere. ${ }^{23}$ This method allows for accurate estimation of the sample absorption and eliminates dependence of spatial properties of emitted light and is usually employed for solid samples and light scattering samples (polymers, gels, and particle dispersions). ${ }^{24,25}$

2. For solution samples an easier method can be used, which compares the UC emission intensity with the intensity of a reference fluorescent dye and is termed as a relative quantum yield measurement. ${ }^{26,27}$

The most important limitation of the first method is self-absorption of the emitted UC photons due to the fact that even for optically thin samples the emitted light can be reabsorbed as the result of multiple reflections inside the sphere. This, in turn, leads to underestimation of $\Phi_{U C .}{ }^{28}$ Recently, Yanai and et al. reported a value of $\Phi_{U C}=36 \%$ for a benchmark TTA-UC system based on platinum(II) octaethylporphyrin (PtOEP) sensitizer and 9,10-diphenylanthracene (DPA) emitter using both the absolute and relative methods. ${ }^{29}$ The authors showed that the reabsorption correction is essential if $\Phi_{U C}$ measurements are performed inside an integrating sphere ( $\Phi_{U C}$ value of $26 \%$ was obtained without such correction).

From a pragmatic perspective, the relative method is less time-consuming and easier to implement than the absolute quantum yield measurement with the integrating sphere. Using the relative method, the quantum yield is calculated from the comparison of the emission intensities for the measured system and a system that has a known quantum yield value using Equation $1 .{ }^{27}$ 


$$
\Phi_{U C}=\Phi_{r e f}\left(\frac{A_{r e f}}{A_{U C}}\right)\left(\frac{E_{U C}}{E_{r e f}}\right)\left(\frac{n_{U C}}{n_{r e f}}\right)^{2}
$$

where $\Phi$ - UC quantum yield $A$ - absorbance, $E$ - integrated luminescence intensity, and $n$ refractive index. The indices $U C$ and $r e f$ denote sample and reference, respectively.

Many prominent reference dyes such as Rhodamine $\mathrm{B}^{30}$, Rhodamine $101^{31}$, Rhodamine $6 \mathrm{G}^{32}$ Methylene Blue ${ }^{19}$, Nile Red ${ }^{33}$ as well as 2,6-diiodo-8-phenyl BODIPY (2I-BODIPY) showing prompt fluorescence have been used as references for the measurement of relative $\Phi_{U C .}{ }^{34}$ However, in all these cases it is very difficult to assess the uncertainty in the reported $\Phi_{U C}$ values. The result of our analysis of possible uncertainties for the absolute and relative methods (Supporting Information) indicates that relative error in the absorption measurements using a spectrophotometer is the major source of uncertainty in the relative method as compared to the absolute method. The relative error of absorption measurements, which is usually not less than $1-3 \%$, can increase up to $15 \%$ under certain experimental circumstances. ${ }^{27,35}$

Thus, to improve the precision of $\Phi_{U C}$ measurements, there is a need for both a new procedure and a new reference system. For instance, an UC system that uses a sensitizer with reliably measured quantum yield of the prompt fluorescence, which is not affected by the emitter molecules or the media, would allow for the measurement of $\Phi_{U C}$ with low uncertainty and pave the way to an UC-based reference. In this scenario, Equation 1 can be greatly simplified to give Equation 2:

$$
\Phi_{U C}=\Phi_{\text {ref }} \frac{E_{U C}}{E_{\text {ref }}}
$$

The $\Phi_{U C}$ value determined using Equation 2 can be further used as an UC-based reference to measure $\Phi_{U C}$ of other UC samples using Equation 1. Moreover, as suggested by Yanai et al. ${ }^{29}$ the UC-based reference exhibiting a prompt sensitizer luminescence can be additionally utilized to verify the measurement setup and presence of oxygen in the UC reference sample. 


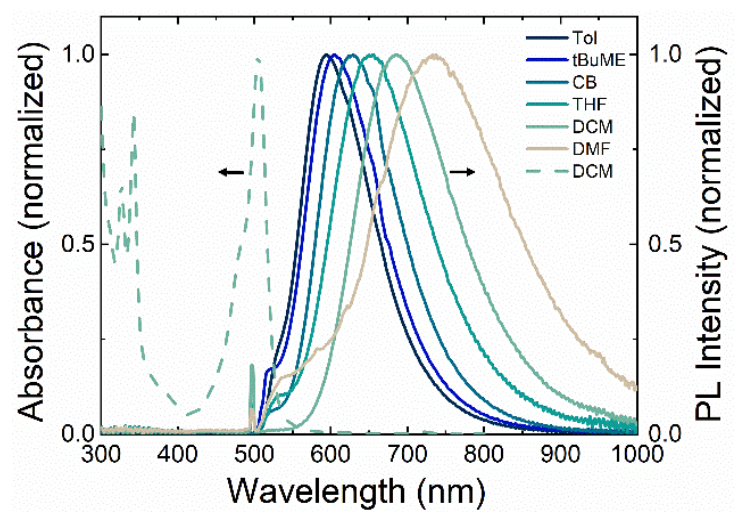

Figure 2. Normalized absorption (dashed line) in DCM and the photoluminescence (PL) spectra of BD1 in deoxygenated solvents (solid lines): toluene (Tol), methyl tert-butyl ether (tBuME), 1-chlorobutane (CB), tetrahydrofuran (THF), dichloromethane (DCM), and N,Ndimethylformamide (DMF). Concentration of BD1 $\left(\mathrm{C}_{\mathrm{BD} 1}\right)$ is $1 \times 10^{-5} \mathrm{M}$, excitation wavelength $\left(\lambda_{\text {exc }}\right)$ is $498 \mathrm{~nm}$.

As a first step toward the UC-based reference system, we synthesized and investigated optical properties of dyad BD1 (Figure 1c). The absorption spectrum of BD1 in DCM (Figure 2) exhibits typical transitions associated with pyrene (300-400 nm) and BODIPY (450-530 nm) subunits, indicating weak coupling of the chromophores in the ground state. The shape and maxima of the absorption bands are almost unchanged in solvents of different polarity (Figure S2). No red-shifted CT absorption bands were observed for this dyad, in contrast to similar BODIPY-phenoxazine ${ }^{36}$ and perylene dyads ${ }^{19}$ reported by Zhao and co-workers. At the same time, the emission spectra of BD1 exhibit a profound effect of solvent polarity on the excitedstate transitions. While in non-polar hexane a typical narrow BODIPY emission band with a maximum at $520 \mathrm{~nm}$ is observed (Figure S3), a broadening and shifting the emission (originating from the CT state) towards the near-infrared region occurs in toluene $(593 \mathrm{~nm})$ and more polar solvents. The values of quantum yield for Stokes fluorescence $\left(\Phi_{\text {ref }}\right)$ and emission maxima obtained in six different solvents are presented in Table S1. The highest $\Phi_{\text {ref }}$ of $22.4 \pm 0.6 \%$ was observed in toluene, whereas the lowest value of $\Phi_{\text {ref }}=0.1 \%$ was measured 
in polar DMF. The effect of solvent polarity on the emission properties evidences the electron transfer process occurring within the dyad and resulting in formation of a highly polar CT state with positively charged donor (pyrene) subunit and negatively charged acceptor (BODIPY) subunit. As previously mentioned, the CT state in BD1 undergoes SOCT-ISC to populate the triplet state of the BODIPY. High triplet state yield in this dyad was confirmed by transient absorption spectroscopy measurements and singlet oxygen sensitization experiments $\left(\Phi_{\Delta}=75\right.$ $\%$ in ethanol). ${ }^{37}$ BODIPY triplet states are known to be poorly emissive and corresponding phosphorescence can be detected only in glassy solvent matrix at low temperatures. ${ }^{38}$ According to the photoluminescence data presented above, another relaxation channel of the CT state is the emission into the ground state. It should be noted that although CT states are generally considered to be dark, recent results have indicated enhanced emissivity of CT states in dyads based on alkyl-unsubstituted BODIPY scaffold, proposed to be the effect of increased electronic coupling magnitude between the electron donor and acceptor subunits. ${ }^{39}$

To examine the photosensitizing ability of BD1 in green-to-blue TTA-UC, the dyad was combined with perylene in different solvents and photoluminescence under excitation with 498 nm light was monitored (Figure 3a). 

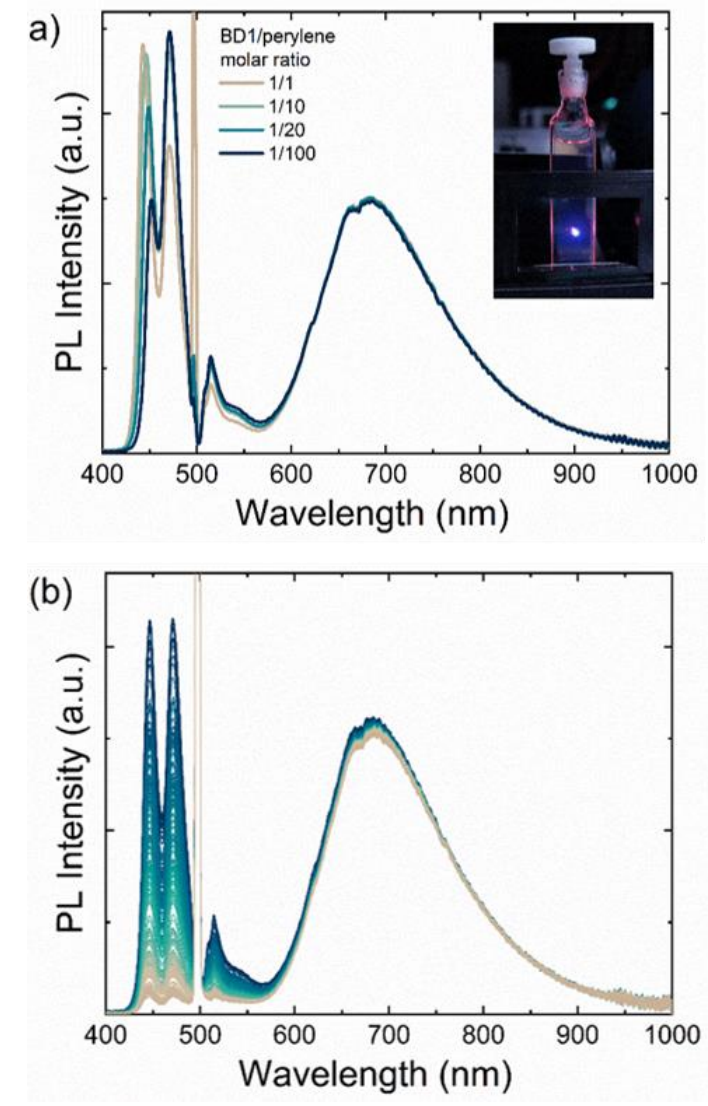

Figure 3. (a) Emission spectra of BD1- perylene mixtures with different molar ratios in deoxygenated DCM. The insert is photograph showing blue UC emission of a BD1-perylene mixture (1:10 molar ratio) excited with $498 \mathrm{~nm}$ laser. The picture was taken with a smartphone camera using no filters.; (b) Emission spectra BD1-perylene mixtures (molar ratio 1:10) at different oxygen content in DCM. Intensity of the UC emission band is gradually decreasing with time (within 25 minutes) due to rising concentration of oxygen in the opened cuvette; Concentration of $\mathrm{BD} 1\left(\mathrm{C}_{\mathrm{BD} 1}\right)$ is $1 \times 10^{-5} \mathrm{M}$, excitation wavelength $\left(\lambda_{\mathrm{exc}}\right)$ is $498 \mathrm{~nm}$, intensity (I) is $5 \mathrm{~W} / \mathrm{cm}^{2}$ for (a) and (b). The peak at $498 \mathrm{~nm}$ in (a) and (b) corresponds to the residual excitation light that passes through a notch optical filter. All shown spectra are raw spectra (without normalization).

The emission observed at 350-450 nm originates from the TTA-UC mechanism and is typical for perylene fluorescence. To estimate $\Phi_{U C}$ values for the BD1 - perylene pair and develop the UC-based reference system, we employed a combination of two methods: relative and absolute 
quantum yield measurements. We assumed that the CT emission of BD1 due to its very large Stokes-shift can be used as a self-reference for the determination of $\Phi_{U C}$. Such approach has two important advantages: (i) absolute quantum yield value ( $\left.\Phi_{r e f}\right)$ for red-shifted CT emission can be estimated with high accuracy using an integrating sphere; and (ii) the CT emission is not masked by UC luminescence coming from perylene, with the two emission bands being very well separated (Figure 3a). Thus, accurate calculation of the relative $\Phi_{U C}$ value can be performed using the absolute value of $\Phi_{\text {ref }}$.

For the UC-based reference system, we chose DCM because in this solvent BD1 exhibits both a large Stokes shift $(\Delta \lambda=181 \mathrm{~nm})$ and an appreciable fluorescence quantum yield of $\Phi_{r e f}=7.0 \pm 0.2 \%$ (the absolute error $\pm 0.2 \%$.was derived based on error analysis presented in the Supporting Information section). For comparison, while in DMF the Stokes shift is even larger $(\Delta \lambda=229 \mathrm{~nm})$, the emission is substantially weaker $\left(\Phi_{r e f}=0.1 \%\right)$, presumably due to higher polarity of the solvent.

To confirm that the CT emission of BD1 is not reabsorbed in the integrating sphere due to its large Stokes shift, we determined quantum yield values for this emission at various concentrations of BD1. We found that the value of $\Phi_{\text {ref }}$ remains almost unchanged for different BD1 concentrations (Table S2).

To prove that the prompt fluorescence of BD1 can be used as the self-reference, we additionally investigated a role of several important parameters - namely emitter concentration, oxygen quenching, and excitation intensity - as well as performing UC measurements both inside and outside an integrating sphere. The UC PL spectra for samples with different concentrations of perylene and fixed concentration of BD1 are shown in Figure 3a. While the UC PL intensity is greatly affected by the concentration of the emitter, no change in the CT emission intensity is observed, indicating that this emission is not quenched by perylene. Importantly, the presence of oxygen also exhibited no influence on BD1 emission band as can be seen from Figure $\mathbf{3 b}$. 
In the corresponding experiment, oxygen-free BD1-perylene solution was prepared inside a glovebox (oxygen concentration of $0.1 \mathrm{ppm}$ ) and, after the first UC measurement, the cuvette was unsealed, thus open to air. Figure $3 \mathbf{b}$ demonstrates a drastic decrease of the UC PL intensity within a 25-minute period due to oxygen diffusion into the sample, however, importantly, the BD1 fluorescence peak remains unchanged. Thus, overall, the CT emission of BD1 is not influenced by any parameters related to $\Phi_{U C}$ measurements (sensitizer and emitter concentrations, oxygen contamination and excitation intensity (the effect of excitation intensity is demonstrated in Figure $\mathbf{S 4 a}$ ) and can be confidently used as the self-reference for measuring $\Phi_{U C}$ via the relative method.

Using the relative method (equation 2), $\Phi_{U C}$ for the UC-based reference based on the BD1perylene pair in DCM (concentrations of $1 \times 10^{-5} \mathrm{M} / 1 \times 10^{-4} \mathrm{M}$, respectively) was estimated to be $\Phi_{U C}=2.5 \pm 0.1 \%$. A significant underestimation of UC quantum yield $\left(\Phi_{U C(\text { sphere })}=\right.$ $1.6 \pm 0.1 \%$ ) was observed if the absolute method was used for the same UC system. As already stated previously by Yanai et al., the absolute measurements of $\Phi_{U C}$ can be strongly affected by re-absorption inside the integrating sphere. For example, spectra measured for the same sample performed inside and outside the integrating sphere are presented in Figure S5. The shape of the spectra clearly uncovers the effect of the re-absorption process, which leads to different values of $\Phi_{U C}$ obtained using the relative and absolute methods.

It was also important to compare the relative $\Phi_{U C}$ measured using the self-reference and a common fluorescence quantum yield standard. Figure S6 displays $\Phi_{U C}$ values BD1-perylene system in DCM (concentrations of $1 \times 10^{-5} \mathrm{M} / 1 \times 10^{-4} \mathrm{M}$, respectively), measured relative to solutions of Rhodamine $6 \mathrm{G}$ with different concentrations (in a range of $5 \times 10^{-7}-10^{-5} \mathrm{M}$ ). These results demonstrate a gradual increase of $\Phi_{U C}$ from $2.3 \%$ to $3.3 \%$ with increasing the concentration of Rhodamine $6 \mathrm{G}$ reference solution. We assume that the highest (overestimated) value of $3.3 \% \%$ can be explained by the decrease in $\Phi_{\text {ref }}$ value due to reabsorption 
ofRhodamine $6 \mathrm{G}$ fluorescence in concentrated solution $\left(10^{-5} \mathrm{M}\right)$. Fore more dilute solutions of Rhodamine 6G, we expect an increase in the uncertainty of the absorption measurements $\left(A_{r e f}\right)$, that, in turn, can lead to underestimation of the quantum yield values.

Before exploiting the newly developed UC-based reference in further experiments, we also applied the reference for estimation of $\Phi_{U C}$ for the benchmark system PtOEP-DPA $\left(1 \times 10^{-}\right.$ ${ }^{4} \mathrm{M} / 1 \times 10^{-2} \mathrm{M}$ in deoxygenated THF, under excitation with 498 light $\mathrm{nm}$ and intensity of 5 $\mathrm{W} / \mathrm{cm}^{2}$ ). Excellent agreement was found between our results, where we obtained $\Phi_{U C}=18.0 \pm 0.4 \%$, and the result reported by Yanai et al. with $2 \times \Phi_{U C}=36 \%$. Thus, we believe that the $\Phi_{U C}$ value estimated with BD1-perylene system is characterized by a very little uncertainty and it can be used as a UC-based reference for characterization of other UC systems.

To demonstrate several examples of application of the developed UC-based reference, we investigated a rough solvent impact on $\Phi_{U C}$ of the BD1-pyrelene pair. It is well-known that solvent can have a profound effect on photochemical reactions, as well as on energy and electron transfer rates. ${ }^{36-37,40-44}$ Using the UC-based reference, we systematically measured $\Phi_{U C}$ of the BD1-perylene pair in solvents of different polarity and viscosity. To correlate $\Phi_{U C}$ with solvent properties, we chose the solvent polarity parameter $\mathrm{E}_{\mathrm{t}}(30)$ - as it is widely used as a parameter reflecting both hydrogen bonding and electrostatic interactions ${ }^{45-46}$ - and solvent viscosity ${ }^{47}$ as another parameter (Table S3). The UC-spectra measured in different solvents are shown in Figure 4a and Figure S7. 

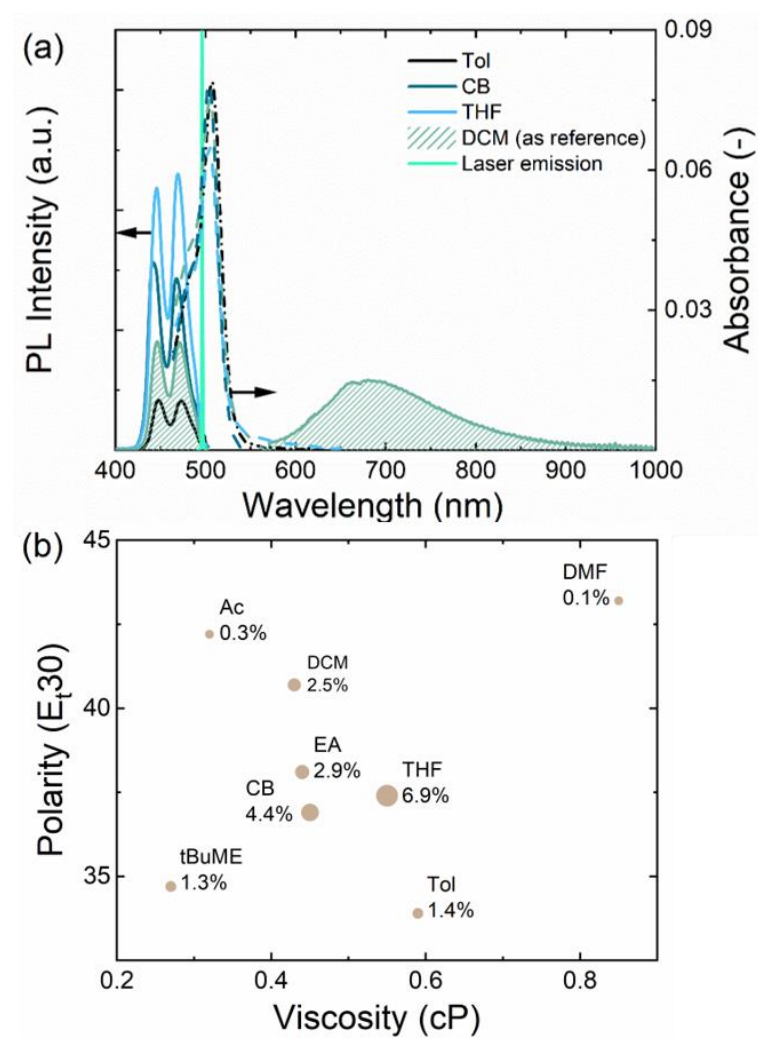

Figure 4. (a) Absorption (dashed lines) and UC PL spectra (solid lines) of the BD1-perylene pair $(1: 10$ molar ratio $)$ in different deoxygenated solvents $\left(\mathrm{C}_{\mathrm{BDP}}=1 \times 10^{-5} \mathrm{M}\right)$. Excitation source is $498 \mathrm{~nm} \mathrm{M} 2$ laser $\left(5 \mathrm{~W} / \mathrm{cm}^{2}\right)$. UC PL of the BD1-perylene pair in deoxygenated DCM (shaded area) was used as a reference for calculation of $\Phi_{\mathrm{UC}}$ in different solvents via the relative method. The CT fluorescence of BD1 with $\Phi_{r e f}=7.0 \%$ is shown for comparison. Full emission spectra in different solvents can be found in Figure S8; (b) $\Phi_{U C}$ values measured for the BD1-perylene pair in solvents of different polarity and viscosity. Viscosity and polarity values of solvents are summarized in Table S3.

The results of $\Phi_{U C}$ measurements in these solvents using both the relative method and the absolute method are presented in Figure $\mathbf{4 b}$ and Table S4. As was mentioned above, the absolute method significantly underestimates UC quantum yield values and, therefore, only $\Phi_{U C}$ obtained with relative method will be discussed further. We arbitrary considered $\Phi_{U C}$ of $1.4 \pm 0.1 \%$ in toluene as a starting point of discussion. The increase of polarity leads to a 
substantial increase of $\Phi_{U C}$ values leading to the highest value of $\Phi_{U C}$ of $6.9 \pm 0.2 \%$ achieved in THF. Interestingly, further increase in solvent polarity does not result in an increase of $\Phi_{U C}$. Values of $0.3 \%$ and $0.1 \%$ were obtained in polar solvents with low and high viscosity, acetone (Ac) and DMF, respectively. This can be explained by the fact that in highly polar solvents the CT state is strongly stabilized, resulting in rapid ground state recombination and low triplet state yields. ${ }^{48}$ As can be seen from Figure $4 \mathbf{b}$, as the viscosity increases from $0.27-0.85 \mathrm{cP}$, there is no clear trend in how this affects the efficiency of the UC process. $\Phi_{U C}$ appears to be comparable for solvents with similar $\mathrm{E}_{\mathrm{t}} 30$ values, but with different viscosity. These results can be explained assuming that either TTET or TTA rate is slower than the maximum rate of a diffusion controlled process (with the expected value of $\sim 2 \times 10^{10} \mathrm{M}^{-1} \mathrm{~s}^{-1}$ ) 49 .

Finally, our calculations of $\Phi_{U C}$ using the UC reference were extended to donor-acceptor dyads BD2 and BD3, bearing anthracene and perylene as donor subunits, respectively (Figure 1c). Corresponding absorption and UC PL spectra are presented in Figures S9 and S10. Similar to BD1 sensitizer, the highest value of $\Phi_{U C}=3.6 \pm 0.1 \%$ (Table 1) for BD2 was obtained in THF (under $498 \mathrm{~nm}$ excitation).

Table 1. Quantum yields of UC-systems based on $B D 2$ and $B D 3\left(\mathrm{C}_{\mathrm{BD}}=1 \times 10^{-5} \mathrm{M}\right.$, sensitizeremitter ratio 1:10) in different solvents, measured by absolute and relative methods. The excitation wavelengths are $525 \mathrm{~nm}\left(5 \mathrm{~W} / \mathrm{cm}^{2}\right)$ and $498 \mathrm{~nm}\left(5 \mathrm{~W} / \mathrm{cm}^{2}\right)$ for BD2 and BD3, respectively.

\begin{tabular}{crrrr} 
Sensitizer & \multicolumn{2}{c}{ BD2 } & \multicolumn{2}{c}{ BD3 } \\
Solvent & THF & DCM & THF & DCM \\
$\boldsymbol{\Phi}_{U C}, \%$ & 3.6 & 2.8 & 1.3 & 1.8 \\
\hline$\Phi_{U C(\text { sphere })}, \%$ & 1.6 & 1.3 & 0.4 & 0.7 \\
\hline
\end{tabular}


In contrast, a system based on the BD3 dyad exhibits the highest efficiency in DCM $\left(\Phi_{U C}=1.8 \pm 0.1 \%\right)$, presumably because the charge transfer process in this dyad requires more polar environment to be efficient. To note, the BD3 dyads demonstrates significant red-shift in absorption spectra as compared with $\mathrm{BD} 1$ and $\mathrm{BD} 2$, so another excitation wavelength $(525 \mathrm{~nm})$ was used for the $\Phi_{U C}$ measurement. It was proved that the reference $\Phi_{U C}$ value $(2.5 \pm 0.1 \%)$ does not change with excitation wavelength (if the excitation intensity exceeds the UC threshold of $1.1 \mathrm{~W} / \mathrm{cm}^{2}$ estimated for $525 \mathrm{~nm}$ excitation). Thus, our reference system can be used for estimation of $\Phi_{U C}$ of green-to-blue UC systems using the relative method in the spectral range of $\sim 450-530 \mathrm{~nm}$ (covered by the BD1 sensitizer).

To conclude, in this work we investigated the properties of BODIPY-pyrene (BD1) donoracceptor dyad, which exhibits near-infrared CT emission in various solvents. The dyad undergoes efficient ISC and enables green-to blue TTA-UC in a pair with perylene as an emitter. We found that the quantum yield of the dyad prompt fluorescence $\left(\Phi_{r e f}\right)$ is unaffected by the parameters of UC system, such as concentrations and ratio of the components, presence of oxygen, and excitation intensity. We demonstrated that the prompt fluorescence of BD1 enables it to be used as the self-reference for the relative method and developed the UC-based reference system based on the BD1-perylene pair. Such approach was found to have less uncertainty in estimation of $\Phi_{U C}$ with respect to the standard method employing a reference fluorescent dye (Rhodamine 6G). At the same time, we found that the BD1-perylene pair displays one of the highest $\Phi_{U C}$ of $6.9 \pm 0.2 \%$ reported for heavy-atom-free sensitizers to date. Using the UC-based reference, we performed accurate measurement of $\Phi_{U C}$ values for several BODIPY-based heavy-atom-free sensitizers in different solvents and compared these results with absolute $\Phi_{U C}$ values measured with an integrating sphere. Due to synthetic accessibility of BD1 and its unique fluorescence behavior, in particular strongly emissive CT state, the 
proposed BD1-perylene system can be broadly applied as UC-based reference for measurements of UC quantum yields under green light excitation.

\section{ASSOCIATED CONTENT}

\section{Supporting Information}

The Supporting Information is available free of charge on the ACS Publications website.

Synthesis of BD1-3, characterization data, experimental procedure for optical measurements, supporting Figure (PDF)

\section{AUTHORS INFORMATION}

Corresponding Authors

*E-mail: andrey.turshatov@kit.edu; mikhail.filatov@tudublin.ie

\section{ORCID}

Bryce S. Richards:0000-0001-5469-048X

Mikhail Filatov: 0000-0002-1640-841X

Andrey Turshatov:0000-0002-8004-098X

\section{ACKNOWLEDGMENT}

B.S.R, and A.T. acknowledge the Helmholtz Energy Materials Foundry (HEMF). N.K. acknowledges a DAAD scholarship. M.F. acknowledges TU Dublin for support of this work.

\section{REFERENCES}

(1) Zhou, J.; Liu, Q.; Feng, W.; Sun, Y.; Li, F., Upconversion Luminescent Materials: Advances and Applications. Chem. Rev. 2015, 115, 395-465.

(2) Choi, D.; Nam, S. K.; Kim, K.; Moon, J. H., Enhanced Photoelectrochemical Water Splitting through Bismuth Vanadate with a Photon Upconversion Luminescent Reflector. Angew. Chem. Int. Ed. 2019, 58, 6891-6895. 
(3) Yu, T.; Liu, Y.; Zeng, Y.; Chen, J.; Yang, G.; Li, Y., Triplet-Triplet Annihilation Upconversion for Photocatalytic Hydrogen Evolution. Chem. Eur. J 2019, 25, 16270-16276.

(4) Ravetz, B. D.; Pun, A. B.; Churchill, E. M.; Congreve, D. N.; Rovis, T.; Campos, L. M., Photoredox catalysis using infrared light via triplet fusion upconversion. Nature 2019, 565, 343-346.

(5) Gray, V.; Dzebo, D.; Abrahamsson, M.; Albinsson, B.; Moth-Poulsen, K., Triplettriplet annihilation photon-upconversion: towards solar energy applications. Phys. Chem. Chem. Phys. 2014, 16, 10345-10352.

(6) Dilbeck, T.; Hanson, K., Molecular Photon Upconversion Solar Cells Using Multilayer Assemblies: Progress and Prospects. J. Phys. Chem. Lett. 2018, 9, 5810-5821.

(7) Dou, Q.; Jiang, L.; Kai, D.; Owh, C.; Loh, X. J., Bioimaging and biodetection assisted with TTA-UC materials. Drug Discov. Today 2017, 22, 1400-1411.

(8) Askes, S. H. C.; Kloz, M.; Bruylants, G.; Kennis, J. T. M.; Bonnet, S., Triplet-triplet annihilation upconversion followed by FRET for the red light activation of a photodissociative ruthenium complex in liposomes. Phys. Chem. Chem. Phys. 2015, 17, 27380-27390.

(9) Zhao, J.; Wu, W.; Sun, J.; Guo, S., Triplet photosensitizers: from molecular design to applications. Chem. Soc. Rev. 2013, 42, 5323-5351.

(10) Marian, C. M., Spin-orbit coupling and intersystem crossing in molecules. WIREs Comput. Mol. Sci. 2012, 2, 187-203.

(11) Wang, Z.; Zhao, J.; Barbon, A.; Toffoletti, A.; Liu, Y.; An, Y.; Xu, L.; Karatay, A.; Yaglioglu, H. G.; Yildiz, E. A.; Hayvali, M., Radical-Enhanced Intersystem Crossing in New Bodipy Derivatives and Application for Efficient Triplet-Triplet Annihilation Upconversion. J. Am. Chem. Soc. 2017, 139, 7831-7842.

(12) Wang, Z.; Gao, Y.; Hussain, M.; Kundu, S.; Rane, V.; Hayvali, M.; Yildiz, E. A.; Zhao, J.; Yaglioglu, H. G.; Das, R.; Luo, L.; Li, J., Efficient Radical-Enhanced Intersystem Crossing in an NDI-TEMPO Dyad: Photophysics, Electron Spin Polarization, and Application in Photodynamic Therapy. Chem. Eur. J 2018, 24, 18663-18675.

(13) Nagarajan, K.; Mallia, A. R.; Muraleedharan, K.; Hariharan, M., Enhanced intersystem crossing in core-twisted aromatics. Chem. Sci. 2017, 8, 1776-1782.

(14) Hou, Y.; Zhang, X.; Chen, K.; Liu, D.; Wang, Z.; Liu, Q.; Zhao, J.; Barbon, A., Charge separation, charge recombination, long-lived charge transfer state formation and intersystem crossing in organic electron donor/acceptor dyads. J. Mater. Chem. C 2019, 7, 12048-12074.

(15) Gibbons, D. J.; Farawar, A.; Mazzella, P.; Leroy-Lhez, S.; Williams, R. M., Making triplets from photo-generated charges: observations, mechanisms and theory. Photochem. Photobiol. Sci. 2020, 19, 136-158.

(16) Dance, Z. E. X.; Mickley, S. M.; Wilson, T. M.; Ricks, A. B.; Scott, A. M.; Ratner, M. A.; Wasielewski, M. R., Intersystem Crossing Mediated by Photoinduced Intramolecular Charge Transfer: Julolidine-Anthracene Molecules with Perpendicular $\pi$ Systems. J. Phys. Chem. A 2008, 112, 4194-4201.

(17) Wang, Z.; Zhao, J., Bodipy-Anthracene Dyads as Triplet Photosensitizers: Effect of Chromophore Orientation on Triplet-State Formation Efficiency and Application in TripletTriplet Annihilation Upconversion. Org. Lett. 2017, 19, 4492-4495.

(18) Kiseleva, N.; Filatov, M. A.; Oldenburg, M.; Busko, D.; Jakoby, M.; Howard, I. A.; Richards, B. S.; Senge, M. O.; Borisov, S. M.; Turshatov, A., The Janus-faced chromophore: a donor-acceptor dyad with dual performance in photon up-conversion. Chem. Commun. 2018, $54,1607-1610$.

(19) Wang, Z.; Zhao, J.; Di Donato, M.; Mazzone, G., Increasing the anti-Stokes shift in TTA upconversion with photosensitizers showing red-shifted spin-allowed charge transfer absorption but a non-compromised triplet state energy level. Chem. Commun. 2019, 55, 15101513 . 
(20) Filatov, M. A., Heavy-atom-free BODIPY photosensitizers with intersystem crossing mediated by intramolecular photoinduced electron transfer. Org. Biomol. Chem. 2020, 18, 1027.

(21) A. D. McNaught, A. W., IUPAC. Compendium of Chemical Terminology. Blackwell Scientific Publications, Oxford 1997.

(22) Gray, V.; Moth-Poulsen, K.; Albinsson, B.; Abrahamsson, M., Towards efficient solidstate triplet-triplet annihilation based photon upconversion: Supramolecular, macromolecular and self-assembled systems. Coord. Chem. Rev. 2018, 362, 54-71.

(23) de Mello, J. C.; Wittmann, H. F.; Friend, R. H., An improved experimental determination of external photoluminescence quantum efficiency. Adv. Mater. 1997, 9, 230232.

(24) Marsico, F.; Turshatov, A.; Peköz, R.; Avlasevich, Y.; Wagner, M.; Weber, K.; Donadio, D.; Landfester, K.; Baluschev, S.; Wurm, F. R., Hyperbranched Unsaturated Polyphosphates as a Protective Matrix for Long-Term Photon Upconversion in Air. J. Am. Chem. Soc. 2014, 136, 11057-11064.

(25) Turshatov, A.; Busko, D.; Kiseleva, N.; Grage, S. L.; Howard, I. A.; Richards, B. S., Room-Temperature High-Efficiency Solid-State Triplet-Triplet Annihilation Up-Conversion in Amorphous Poly(olefin sulfone)s. ACS Appl. Mater. Interfaces 2017, 9, 8280-8286.

(26) Crosby, G. A.; Demas, J. N., Measurement of photoluminescence quantum yields. Review. J. Phys. Chem. 1971, 75, 991-1024.

(27) Würth, C.; Grabolle, M.; Pauli, J.; Spieles, M.; Resch-Genger, U., Relative and absolute determination of fluorescence quantum yields of transparent samples. Nat. Protoc. 2013, 8, 1535-1550.

(28) Ahn, T.-S.; Al-Kaysi, R. O.; Müller, A. M.; Wentz, K. M.; Bardeen, C. J., Selfabsorption correction for solid-state photoluminescence quantum yields obtained from integrating sphere measurements. Rev. Sci. Instrum 2007, 78, 086105.

(29) Yanai, N.; Suzuki, K.; Ogawa, T.; Sasaki, Y.; Harada, N.; Kimizuka, N., Absolute Method to Certify Quantum Yields of Photon Upconversion via Triplet-Triplet Annihilation. J. Phys. Chem. A 2019, 123, 10197-10203.

(30) Liu, Q.; Yang, T.; Feng, W.; Li, F., Blue-Emissive Upconversion Nanoparticles for Low-Power-Excited Bioimaging in Vivo. J. Am. Chem. Soc. 2012, 134, 5390-5397.

(31) Vadrucci, R.; Weder, C.; Simon, Y. C., Organogels for low-power light upconversion. Mater. Horiz. 2015, 2, 120-124.

(32) Wei, D.; Ni, F.; Zhu, Z.; Zou, Y.; Yang, C., A red thermally activated delayed fluorescence material as a triplet sensitizer for triplet-triplet annihilation up-conversion with high efficiency and low energy loss. J. Mater. Chem. C 2017, 5, 12674-12677.

(33) Hisamitsu, S.; Yanai, N.; Kimizuka, N., Photon-Upconverting Ionic Liquids: Effective Triplet Energy Migration in Contiguous Ionic Chromophore Arrays. Angew. Chem. Int. Ed. 2015, 54, 11550-11554.

(34) Zhou, Q.; Zhou, M.; Wei, Y.; Zhou, X.; Liu, S.; Zhang, S.; Zhang, B., Solvent effects on the triplet-triplet annihilation upconversion of diiodo-Bodipy and perylene. Phys. Chem. Chem. Phys. 2017, 19, 1516-1525.

(35) Sooväli, L.; Rõõm, E.-I.; Kütt, A.; Kaljurand, I.; Leito, I., Uncertainty sources in UVVis spectrophotometric measurement. ACCREDIT QUAL ASSUR 2006, 11, 246-255.

(36) Dong, Y.; Sukhanov, A. A.; Zhao, J.; Elmali, A.; Li, X.; Dick, B.; Karatay, A.; Voronkova, V. K., Spin-Orbit Charge-Transfer Intersystem Crossing (SOCT-ISC) in BodipyPhenoxazine Dyads: Effect of Chromophore Orientation and Conformation Restriction on the Photophysical Properties. J. Phys. Chem. C 2019, 123, 22793-22811.

(37) Filatov, M. A.; Karuthedath, S.; Polestshuk, P. M.; Callaghan, S.; Flanagan, K. J.; Wiesner, T.; Laquai, F.; Senge, M. O., BODIPY-Pyrene and Perylene Dyads as Heavy-AtomFree Singlet Oxygen Sensitizers. ChemPhotoChem 2018, 2, 606-615. 
(38) Zhang, X.-F.; Yang, X.; Niu, K.; Geng, H., Phosphorescence of BODIPY dyes. J. Photochem. Photobiol. A 2014, 285, 16-20.

(39) Hou, Y.; Kurganskii, I.; Elmali, A.; Zhang, H.; Gao, Y.; Lv, L.; Zhao, J.; Karatay, A.; Luo, L.; Fedin, M., Electronic coupling and spin-orbit charge transfer intersystem crossing (SOCT-ISC) in compact BDP-carbazole dyads with different mutual orientations of the electron donor and acceptor. J. Chem. Phys 2020, 152, 114701.

(40) Berlman, I. B., Handbook of Fluorescence Spectra of Aromatic Molecules. Academic 1971.

(41) Buck, J. T.; Boudreau, A. M.; DeCarmine, A.; Wilson, R. W.; Hampsey, J.; Mani, T., Spin-Allowed Transitions Control the Formation of Triplet Excited States in Orthogonal Donor-Acceptor Dyads. Chem 2019, 5, 138-155.

(42) Chen, K.; Yang, W.; Wang, Z.; Iagatti, A.; Bussotti, L.; Foggi, P.; Ji, W.; Zhao, J.; Di Donato, M., Triplet Excited State of BODIPY Accessed by Charge Recombination and Its Application in Triplet-Triplet Annihilation Upconversion. J. Phys. Chem. A 2017, 121, 75507564.

(43) Liu, Y.; Zhao, J.; Iagatti, A.; Bussotti, L.; Foggi, P.; Castellucci, E.; Di Donato, M.; Han, K.-L., A Revisit to the Orthogonal Bodipy Dimers: Experimental Evidence for the Symmetry Breaking Charge Transfer-Induced Intersystem Crossing. J. Phys. Chem. C 2018, 122, 2502-2511.

(44) Börjesson, K.; Rudquist, P.; Gray, V.; Moth-Poulsen, K., Photon upconversion with directed emission. Nat. Commun. 2016, 7, 12689.

(45) Reichardt, C., Solvatochromic Dyes as Solvent Polarity Indicators. Chem. Rev. 1994, 94, 2319-2358.

(46) Cerón-Carrasco, J. P.; Jacquemin, D.; Laurence, C.; Planchat, A.; Reichardt, C.; Sraïdi, K., Solvent polarity scales: determination of new ET(30) values for 84 organic solvents. J. Phys. Org. Chem. 2014, 27, 512-518.

(47) Smallwood, I. M., Handbook of Organic Solvent Properties. Elsevier 1996.

(48) Wang, Z.; Ivanov, M.; Gao, Y.; Bussotti, L.; Foggi, P.; Zhang, H.; Russo, N.; Dick, B.; Zhao, J.; Di Donato, M.; Mazzone, G.; Luo, L.; Fedin, M., Spin-Orbit Charge-Transfer Intersystem Crossing (ISC) in Compact Electron Donor-Acceptor Dyads: ISC Mechanism and Application as Novel and Potent Photodynamic Therapy Reagents. Chem. Eur. J 2020, 26, 1091-1102.

(49) Avila, V.; Previtali, C. M.; Chesta, C. A., Free energy dependence of the diffusionlimited quenching rate constants in photoinduced electron transfer processes. Photochem. Photobiol. Sci. 2008, 7, 104-108. 